\section{Drs. Sheth and Aggarwal reply}

\section{To the Editor:}

We were delighted to read the letter by Thakran, et $a l^{1}$ and learn of the different interventions toward the same goals. It is impressive that the use of specialist rheumatology nurses improved the vaccination rate to $52 \%$, which is similar to our results of $51.7 \%^{2}$. Specialist nurses can be helpful in clinical rheumatology practice.

In general, we agree with Thakran, et $a l^{1}$ regarding the risk of developing herpes zoster (HZ) with medications and their intervention of intense counseling, clarifying misconceptions, and emphasizing the importance of $\mathrm{HZ}$ vaccine by a specialty nurse. Such interventions are effective, but not sustainable or affordable to many institutes in the United States and probably around the world.

Currently in the United States, there is a significant shortage of nurses, including specialist rheumatology nurses ${ }^{3}$. For example, in our large practice of 12 rheumatologists (with different clinical effort, 20\%-80\%) and 6 rheumatology fellows, we have only 3 nurses handling a large volume of phone calls a day for disease- or medication-related problems. In addition, they have to process a large number of medication refills, and authorizations for medications and procedures. They also have other educational and patient care responsibilities, such as helping with joint injection/aspiration, self-injection teaching, etc. There is simply no time left for nurses to check a patient's previous vaccination record, interact with the patient regarding vaccination, and then counsel and vaccinate eligible patients, followed by documenting of vaccination. We expect that the scenario is similar in other rheumatology practices across the United States.

Our interventions of electronic identification and best practice alert in electronic medical records have the advantage of self-sustainability for the long term with minimal effort from the staff and physician. Our electronic record system also verifies prior documentation of vaccination and alerts a medical assistant only for the eligible patient based on predetermined, nationally recommended criteria of age and the risk of $\mathrm{HZ}$ disease. In the United States, vaccinations are primarily the responsibility of the primary care doctor. Therefore, for specialty clinics, electronic record-based automated alert queries the system for prior vaccination and eligibility, and it works better in our scenario to use medical assistants for patient education and prescribing vaccination and to involve the physician only for the final review. It is impractical in our practice for a nurse to screen, determine eligibility, and counsel every patient for vaccination.

There are other process issues in the United States that may not be relevant to other countries, such as an insurance coverage check for vaccination. Some insurance plans require vaccines to be prescribed from the primary care doctor and not the specialist.

Thus, both our and the specialty resource nurse interventions as described by Thakran, et al ${ }^{1}$ are effective in improving $\mathrm{HZ}$ vaccination rates. However, our interventions are generalizable to all institutes with electronic medical record systems, and are practical, cost-effective, and self-sustainable with periodic education of providers and orientation of new hired staff. In case of change in vaccine recommendation criteria, electronic alerts can easily be revised to accommodate the change. For areas of the world where nursing resources are easily available and affordable, and for the institutes with no electronic medical records, specialty resource nurses may be an option.

HEENA SHETH, MD, MPH, Department of Medicine, University of Pittsburgh School of Medicine; ROHIT AGGARWAL, MD, MS, Rheumatology Division, Department of Medicine, University of Pittsburgh Medical Center, Pittsburgh, Pennsylvania, USA. Address correspondence to Dr. H. Sheth, University of Pittsburgh, Medicine, 3504 Fifth Ave., Suite 200, Pittsburgh, Pennsylvania 15217, USA.

E-mail: hss2@pitt.edu

\section{REFERENCES}

1. Thakran RD, Baghel SS, Rawat R, Messi C. Herpes zoster vaccination compliance: the role of specialist rheumatology nurses. J Rheumatol 2017;44:960-1.

2. Sheth H, Moreland L, Peterson H, Aggarwal R. Improvement in herpes zoster vaccination in patients with rheumatoid arthritis: a quality improvement project. J Rheumatol 2017;44:11-7.

3. Rosseter R. Nursing shortage fact sheet. [Internet. Accessed March 27, 2017.] Washington: American Association of Colleges of Nursing; 2014. Available from: www.aacn.nche.edu/ media-relations/fact-sheets/nursing-shortage

J Rheumatol 2017;44:6; doi:10.3899/jrheum.170329 